\title{
Stabilization and hybrid synchronization via the adaptive control method of non-identical 4-D financial hyper-chaotic systems
}

Hannachi Fareh

University Larbi Tebessi, Tebessa, Algeria

Received: 13 June 2021, Accepted: 16 June 2021

Published online: 12 July 2021.

\begin{abstract}
In this work, we have considered the stabilization and the hybrid synchronization by the adaptive control method for two non-identical 4-D financial hyper-chaotic systems with unknown parameters, based on Lyapunov's stability theory and the theory of adaptive control. We derive new control results via adaptive control method based on Lyapunov stability theory and adaptive control theory for globally synchronizing two non-identical 4-D financial hyper-chaotic systems with unknown system parameters. The results are validated by numerical simulation using Matlab.
\end{abstract}

Keywords: 4-D financial hyper-chaotic system, Lyapunov exponent, stabilization, synchronization.

\section{Description and stabilization of a new hyper-chatic system}

We consider the financial hyperchaotic system described by the following differential equation model:

$$
\left\{\begin{array}{l}
\frac{d x_{1}}{d t}=-a\left(x_{2}+x_{1}\right)+x_{4} \\
\frac{d x_{2}}{d t}=-x_{2}-a x_{1} x_{3} \\
\frac{d x_{3}}{d t}=b+a x_{1} x_{2} \\
\frac{d x_{4}}{d t}=-d x_{4}-c x_{1} x_{3}
\end{array}\right.
$$

such that $x_{1}, x_{2}, x_{3}, x_{4}$ are the state variables and $a, b, c, d$ are the system parameters respectively. For $a=3 ; b=15$; $c=0,2$ and $d=0,12$, the Lyapunov exponents of the system (1) are:

$$
L_{1}=0.7083, L_{2}=0.032247, L_{3}=0, L_{4}=-4.7139
$$

So, the system (1) has two positive Lyapunov exponents $L_{1}$ et $L_{2}$, which shows that the system is hyperchaotic and the Kaplan-york dimension for this hyperchaotic system is calculated as:

$$
D_{K Y}=3+\frac{L_{1}+L_{2}+L_{3}}{\left|L_{4}\right|}=3.15709
$$

\subsection{Stabiliziting hyperchaotic system (1) via adaptive control:}

In this section, we will build an adaptive controller to globally stabilize the new hyperchaotic system (1), with undefined parameters.

\footnotetext{
* Corresponding author e-mail: fareh.hannachi@univ-tebessa.dz
} 
The controlled hyperchaotic system of system (1) is:

$$
\left\{\begin{array}{l}
\frac{d x_{1}}{d t}=-a\left(x_{2}+x_{1}\right)+x_{4}+u_{1} \\
\frac{d x_{2}}{d t}=-x_{2}-a x_{1} x_{3}+u_{2} \\
\frac{d x_{3}}{d t}=b+a x_{1} x_{2}+u_{3} \\
\frac{d x_{4}}{d t}=-d x_{4}-c x_{1} x_{3}+u_{4}
\end{array}\right.
$$

tel que $x_{1}, x_{2}, x_{3}, x_{4}$ the state variables, $a, b, c, d$ are constants and unknown parameters and $u_{1}, u_{2}, u_{3}, u_{4}$ are adaptive controls to be designed using estimates $\hat{a}(t), \hat{b}(t), \hat{c}(t)$ et $\hat{d}(t)$ which are unknown parameters.

Consider the adaptive controller defined as follows:

$$
\left\{\begin{array}{l}
u_{1}(t)=\hat{a}(t)\left(x_{2}+x_{1}\right)-x_{4}-k_{1} x_{1} \\
u_{2}(t)=x_{2}+\hat{a}(t) x_{1} x_{3}-k_{2} x_{2} \\
u_{3}(t)=-\hat{b}(t)-\hat{a}(t) x_{1} x_{2}-k_{3} x_{3} \\
u_{4}(t)=\hat{d}(t) x_{4}+\hat{c}(t) x_{1} x_{3}-k_{4} x_{4}
\end{array}\right.
$$

where $k_{1}, k_{2}, k_{3}, k_{4}$, are positive constants and $\hat{a}(t), \hat{b}(t), \hat{c}(t), \hat{d}(t)$ are the undefined parameters $a, b, c, d$ respectively.

Introducing (5) into (4), we get:

$$
\left\{\begin{array}{l}
\frac{d x_{1}}{d t}=-(a-\hat{a}(t))\left(x_{2}+x_{1}\right)-k_{1} x_{1} \\
\frac{d x_{2}}{d t}=-(a-\hat{a}(t)) x_{1} x_{3}-k_{2} x_{2} \\
\frac{d x_{3}}{d t}=b-\hat{b}(t)+(a-\hat{a}(t)) x_{1} x_{2}-k_{3} x_{3} \\
\frac{d x_{4}}{d t}=-(d-\hat{d}(t)) x_{4}-(c-\hat{c}(t)) x_{1} x_{3}-k_{4} x_{4}
\end{array}\right.
$$

Now, let's define the error estimation parameters as:

$$
\left\{\begin{array}{l}
e_{a}(t)=a-\hat{a}(t) \\
e_{b}(t)=b-\hat{b}(t) \\
e_{c}(t)=c-\hat{c}(t) \\
e_{d}(t)=d-\hat{d}(t)
\end{array}\right.
$$

using (7), then introducing in (6), and from the simplification we obtain the following equations:

$$
\left\{\begin{array}{l}
\frac{d x_{1}}{d t}=-e_{a}(t)\left(x_{2}+x_{1}\right)-k_{1} x_{1} \\
\frac{d x_{2}}{d t}=-e_{a}(t) x_{1} x_{3}-k_{2} x_{2} \\
\frac{d x_{3}}{d t}=e_{b}(t)+e_{a}(t) x_{1} x_{2}-k_{3} x_{3} \\
\frac{d x_{4}}{d t}=-e_{d}(t) x_{4}-e_{c}(t) x_{1} x_{3}-k_{4} x_{4}
\end{array}\right.
$$

Deffirentiating (7) with respect to $t$ and we obtain:

$$
\left\{\begin{array}{l}
\frac{d e_{a}(t)}{d t}=-\frac{d \hat{a}(t)}{d t} \\
\frac{d e_{b}(t)}{d t}=-\frac{d \hat{b}(t)}{d t} \\
\frac{d e_{c}(t)}{d t}=-\frac{d \hat{c}(t)}{d t} \\
\frac{d e_{d}(t)}{d t}=-\frac{d \hat{d}(t)}{d t}
\end{array}\right.
$$


We use Lyapunov's stability theory to find a law for updating the parameters of the estimates.

Consider a quadratic Lyapunov function given by:

$$
V=1 / 2\left(x_{1}^{2}+x_{2}^{2}+x_{3}^{2}+x_{4}^{2}+e_{a}^{2}+e_{b}^{2}+e_{c}^{2}+e_{d}^{2}\right)
$$

Deffirentiating $V$ along the trajectories of systems (8) and (9) and introducing into $\dot{V}$, we obtain:

$$
\begin{aligned}
\dot{V}= & -k_{1} x_{1}^{2}-k_{2} x_{2}^{2}-k_{3} x_{3}^{2}-k_{4} x_{4}^{2}+e_{a}\left[-\left(x_{2}+x_{1}\right) x_{1}-\frac{d \hat{a}(t)}{d t}\right]+e_{b}\left[x_{3}-\frac{d \hat{b}(t)}{d t}\right] \\
& +e_{c}\left[-x_{1} x_{3} x_{4}-\frac{d \hat{c}(t)}{d t}\right]+e_{d}\left[-x_{4}^{2}-\frac{d \hat{d}(t)}{d t}\right]
\end{aligned}
$$

Then from (11), we take:

$$
\left\{\begin{array}{l}
\frac{d \hat{a}(t)}{d t}=-\left(x_{2}+x_{1}\right) x_{1} \\
\frac{d \hat{b}(t)}{d t}=x_{3} \\
\frac{d \hat{c}(t)}{d t}=-x_{1} x_{3} x_{4} \\
\frac{d \hat{b}(t)}{d t}=-x_{4}^{2}
\end{array}\right.
$$

then;

$$
\dot{V}=\sum_{i=1}^{4}-k_{i} x_{i}^{2}
$$

The hyperchaotic system (1) is globally stabilized by the adaptive control law (5) and the updating law (12), such that $k_{1}, k_{2}, k_{3}, k_{4}$, are positive constants.

\section{Hybrid synchronization of non-identical hyper-chaotic systems}

For this synchronization, as a master system, we choose the financial hyper-chaotic system

$$
\left\{\begin{array}{l}
\dot{x_{1}}=x_{3}+\left(x_{2}-a\right) x_{1}+x_{4} \\
\dot{x_{2}}=1-b x_{2}-x_{1}^{2} \\
\dot{x_{3}}=-x_{1}-c x_{3} \\
\dot{x_{4}}=-d x_{1} x_{2}-k x_{4}
\end{array}\right.
$$

and the slave system we used is the controlled hyperchaotic system of system (1) given by:

$$
\left\{\begin{array}{l}
\frac{d y_{1}}{d t}=-\alpha\left(y_{2}+y_{1}\right)+y_{4}+u_{1} \\
\frac{d y_{2}}{d t}=-y_{2}-\alpha y_{1} y_{3}+u_{2} \\
\frac{d y_{3}}{d t}=\beta+\alpha y_{1} y_{2}+u_{3} \\
\frac{d y_{4}}{d t}=-\delta y_{4}-\gamma y_{1} y_{3}+u_{4}
\end{array}\right.
$$

with $\alpha, \beta, \gamma, \delta$ are the parameters of the system. The error of hybrid synchronization between the two hyper-chaotic systems (14) and (15) is:

$$
\left\{\begin{array}{l}
e_{1}=y_{1}-x_{1} \\
e_{2}=y_{2}+x_{2} \\
e_{3}=y_{3}-x_{3} \\
e_{4}=y_{4}+x_{4}
\end{array}\right.
$$




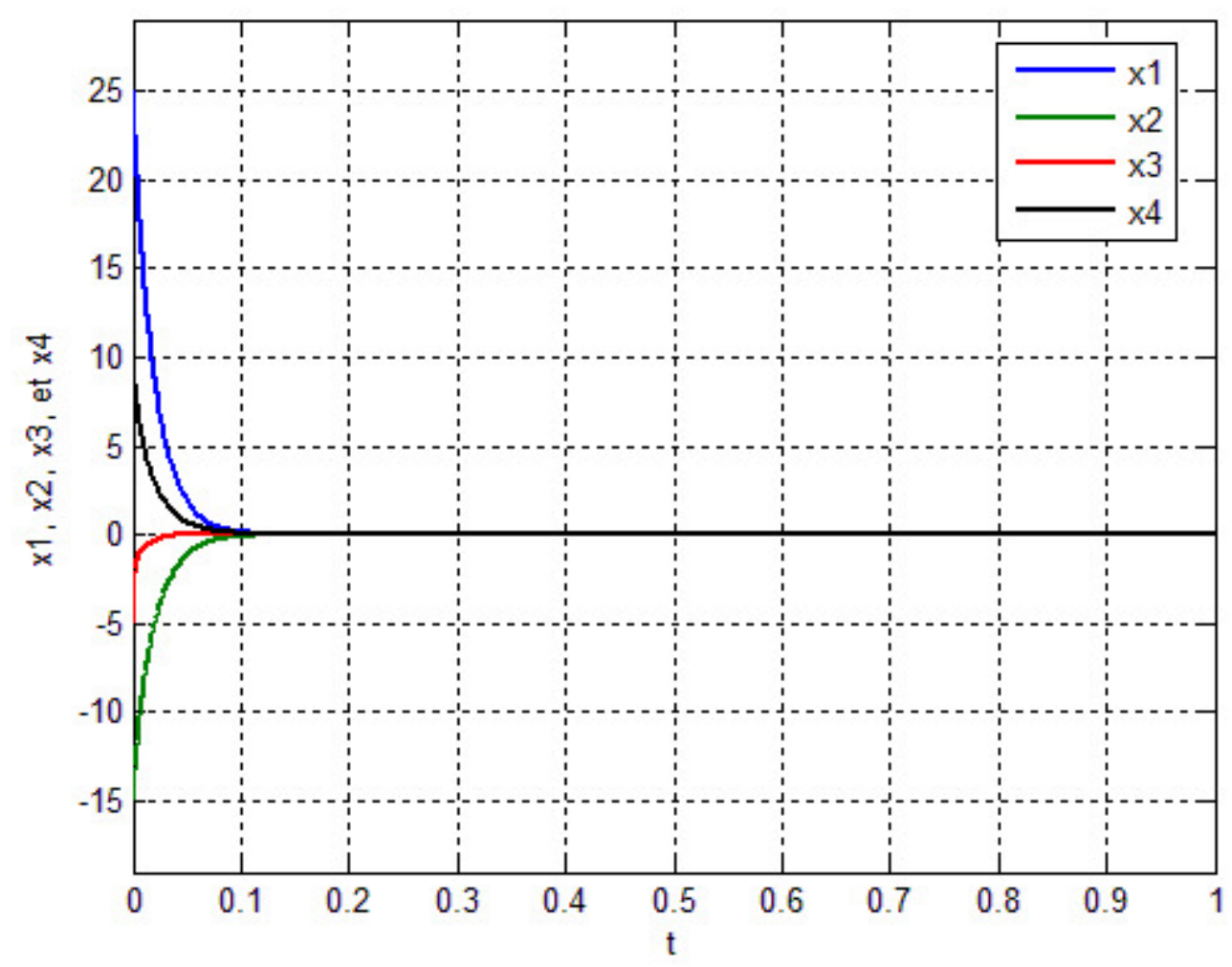

Fig. 1: History of the controlled states $x_{1}, x_{2}, x_{3}, x_{4}$ of the system (1).

then

$$
\left\{\begin{array}{l}
\dot{e}_{1}=\dot{y}_{1}-\dot{x}_{1} \\
\dot{e}_{2}=\dot{y}_{2}+\dot{x}_{2} \\
\dot{e}_{3}=\dot{y}_{3}-\dot{x}_{3} \\
\dot{e}_{4}=\dot{y}_{4}+\dot{x}_{4}
\end{array}\right.
$$

Then the dynamic error of hybrid synchronization is:

$$
\left\{\begin{array}{l}
\dot{e}_{1}=-\alpha\left(y_{2}+y_{1}\right)+y_{4}-\left(x_{3}+\left(x_{2}-a\right) x_{1}+x_{4}\right)+u_{1} \\
\dot{e}_{2}=-y_{2}-\alpha y_{1} y_{3}+\left(1-b x_{2}-x_{1}^{2}\right)+u_{2} \\
\dot{e}_{3}=\beta+\alpha y_{1} y_{2}-\left(-x_{1}-c x_{3}\right)+u_{3} \\
\dot{e}_{4}=-\delta y_{4}-\gamma y_{1} y_{3}+\left(-d x_{1} x_{2}-k x_{4}\right)+u_{4}
\end{array}\right.
$$

Consider the law of adaptive control:

$$
\left\{\begin{array}{l}
u_{1}(t)=\hat{\alpha}(t)\left(y_{2}+y_{1}\right)-y_{4}+\left(x_{3}+\left(x_{2}-\hat{a}(t)\right) x_{1}+x_{4}\right)-k_{1} e_{1} \\
u_{2}(t)=y_{2}+\hat{\alpha}(t) y_{1} y_{3}-\left(1-\hat{b}(t) x_{2}-x_{1}^{2}\right)-k_{2} e_{2} \\
u_{3}(t)=-\hat{\beta}(t)-\hat{\alpha}(t) y_{1} y_{2}+\left(-x_{1}-\hat{c}(t) x_{3}\right)-k_{3} e_{3} \\
u_{4}(t)=\hat{\delta}(t) y_{4}+\hat{\gamma}(t) y_{1} y_{3}-\left(-\hat{d}(t) x_{1} x_{2}-\hat{k}(t) x_{4}\right)-k_{4} e_{4}
\end{array}\right.
$$


Putting (19) in (18), the system (18) becomes:

$$
\left\{\begin{array}{l}
\dot{e}_{1}=-(\alpha-\hat{\alpha})\left(y_{2}+y_{1}\right)+(a-\hat{a}(t)) x_{1}-k_{1} e_{1} \\
\dot{e}_{2}=-(\alpha-\hat{\alpha}(t)) y_{1} y_{3}-(b-\hat{b}(t)) x_{2}-k_{2} e_{2} \\
\dot{e}_{3}=(\beta-\hat{\beta}(t))+(\alpha-\hat{\alpha}(t)) y_{1} y_{2}+(c-\hat{c}(t)) x_{3}-k_{3} e_{3} \\
\dot{e}_{4}=-(\delta-\hat{\delta}(t)) y_{4}-(\gamma-\hat{\gamma}(t)) y_{1} y_{3}-(d-\hat{d}(t)) x_{1} x_{2}-(k-\hat{k}) x_{4}-k_{4} e_{4}
\end{array}\right.
$$

Then the parameters of the error estimates are:

$$
\left\{\begin{array}{l}
e_{\alpha}=(\alpha-\hat{\alpha}) ; e_{\delta}=(\delta-\hat{\delta}) ; e_{\beta}=(\beta-\hat{\beta}) ; e_{\theta}=(\gamma-\hat{\gamma}) \\
e_{a}=(a-\hat{a}) ; e_{b}=(b-\hat{b}) ; e_{c}=(c-\hat{c}) ; e_{d}=(d-\hat{d}) ; e_{k}=(k-\hat{k})
\end{array}\right.
$$

it implies that:

$$
\left\{\begin{array}{l}
\frac{d e_{\alpha}}{d t}=-\frac{d \hat{\alpha}}{d t} ; \frac{d e_{\delta}}{d t}=-\frac{d \hat{\delta}}{d t} ; \frac{d e_{\beta}}{d t}=-\frac{d \hat{\beta}}{d t} ; \frac{d e_{\gamma}}{d t}=-\frac{d \hat{\gamma}}{d t} \\
\frac{d e_{a}}{d t}=-\frac{d \hat{a}}{d t} ; \frac{d e b}{d t}=-\frac{d \hat{b}}{d t} ; \frac{d e_{c}}{d t}=-\frac{d \hat{c}}{d t} ; \frac{d e_{d}}{d t}=-\frac{d \hat{d}}{d t} ; \frac{d e_{k}}{d t}=-\frac{d \hat{k}}{d t}
\end{array}\right.
$$

Replacing (21) in (20), so the dynamic error becomes:

$$
\left\{\begin{array}{l}
\dot{e}_{1}=-e_{\alpha}\left(y_{2}+y_{1}\right)+e_{a} x_{1}-k_{1} e_{1} \\
\dot{e}_{2}=-e_{\alpha} y_{1} y_{3}-e_{b} x_{2}-k_{2} e_{2} \\
\dot{e}_{3}=e_{\beta}+e_{\alpha} y_{1} y_{2}+e_{c} x_{3}-k_{3} e_{3} \\
\dot{e}_{4}=-e_{\delta} y_{4}-e_{\gamma} y_{1} y_{3}-e_{d} x_{1} x_{2}-e_{k} x_{4}-k_{4} e_{4}
\end{array}\right.
$$

Consider the Lyapunov function:

$$
V=\frac{\left(e_{1}^{2}+e_{2}^{2}+e_{3}^{2}+e_{4}^{2}+e_{a}^{2}+e_{b}^{2}+e_{c}^{2}+e_{d}^{2}+e_{\alpha}^{2}+e_{\beta}^{2}+e_{\delta}^{2}+e_{\gamma}^{2}+e_{k}^{2}\right)}{2}
$$

$V$ is a positive definite function in $\mathbb{R}^{13}$. Differentiating the function of Lyapunov $V$ and obtaining the following result:

$$
\left\{\begin{array}{l}
\dot{V}=-k_{1} e_{1}^{2}-k_{2} e_{2}^{2}-k_{3} e_{3}^{2}-k_{4} e_{4}^{2}+e_{a}\left[x_{1} e_{1}-\frac{d \hat{a}}{d t}\right] \\
+e_{b}\left[-x_{2} e_{2}-\frac{d \hat{b}}{d t}\right]+e_{c}\left[x_{3} e_{3}-\frac{d \hat{c}}{d t}\right]+e_{d}\left[-x_{1} x_{2} e_{4}-\frac{d \hat{d}}{d t}\right]+e_{\alpha}\left[-\left(y_{2}+y_{1}\right) e_{1}-y_{1} y_{3} e_{2}+y_{1} y_{2} e_{3}-\frac{d \hat{\alpha}}{d t}\right] \\
+e_{\beta}\left[e_{3}-\frac{d \hat{\beta}}{d t}\right]+e_{\delta}\left[-y_{4} e_{4}-\frac{d \hat{\delta}}{d t}\right]+e_{\gamma}\left[-y_{1} y_{3} e_{4}-\frac{d \hat{\gamma}}{d t}\right]+e_{k}\left[-x_{4} e_{4}-\frac{d \hat{k}}{d t}\right] .
\end{array}\right.
$$

From (25), we take the parameters of the updating law as follows:

$$
\left\{\begin{array}{l}
\frac{d \hat{a}}{d t}=x_{1} e_{1} \\
\frac{d \hat{b}}{d t}=-x_{2} e_{2} \\
\frac{d \hat{c}}{d t}=x_{3} e_{3} \\
\frac{d \hat{d}}{d t}=-x_{1} x_{2} e_{4} \\
\frac{d \hat{\alpha}}{d t}=-\left(y_{2}+y_{1}\right) e_{1}-y_{1} y_{3} e_{2}+y_{1} y_{2} e_{3} \\
\frac{d \hat{\beta}}{d t}=e_{3} \\
\frac{d \hat{\delta}}{d t}=-y_{4} e_{4} \\
\frac{d \hat{\gamma}}{d t}=-y_{1} y_{3} e_{4} \\
\frac{d \hat{k}}{d t}=-x_{4} e_{4}
\end{array}\right.
$$


Replacing (26) in (25), the function $\dot{V}$ become:

$$
\dot{V}=-k_{1} e_{1}^{2}-k_{2} e_{2}^{2}-k_{3} e_{3}^{2}-k_{4} e_{4}^{2}
$$

$\dot{V}$ is a negative definite function in $\mathbb{R}^{4}$. Therefore, we have proved the following result.

Theorem 1. The non-identical hyperchaotic systems (14), (15) with unknown parameters are globally synchronized by the adaptive control law (19), and the updating law for the estimation parameters (26).

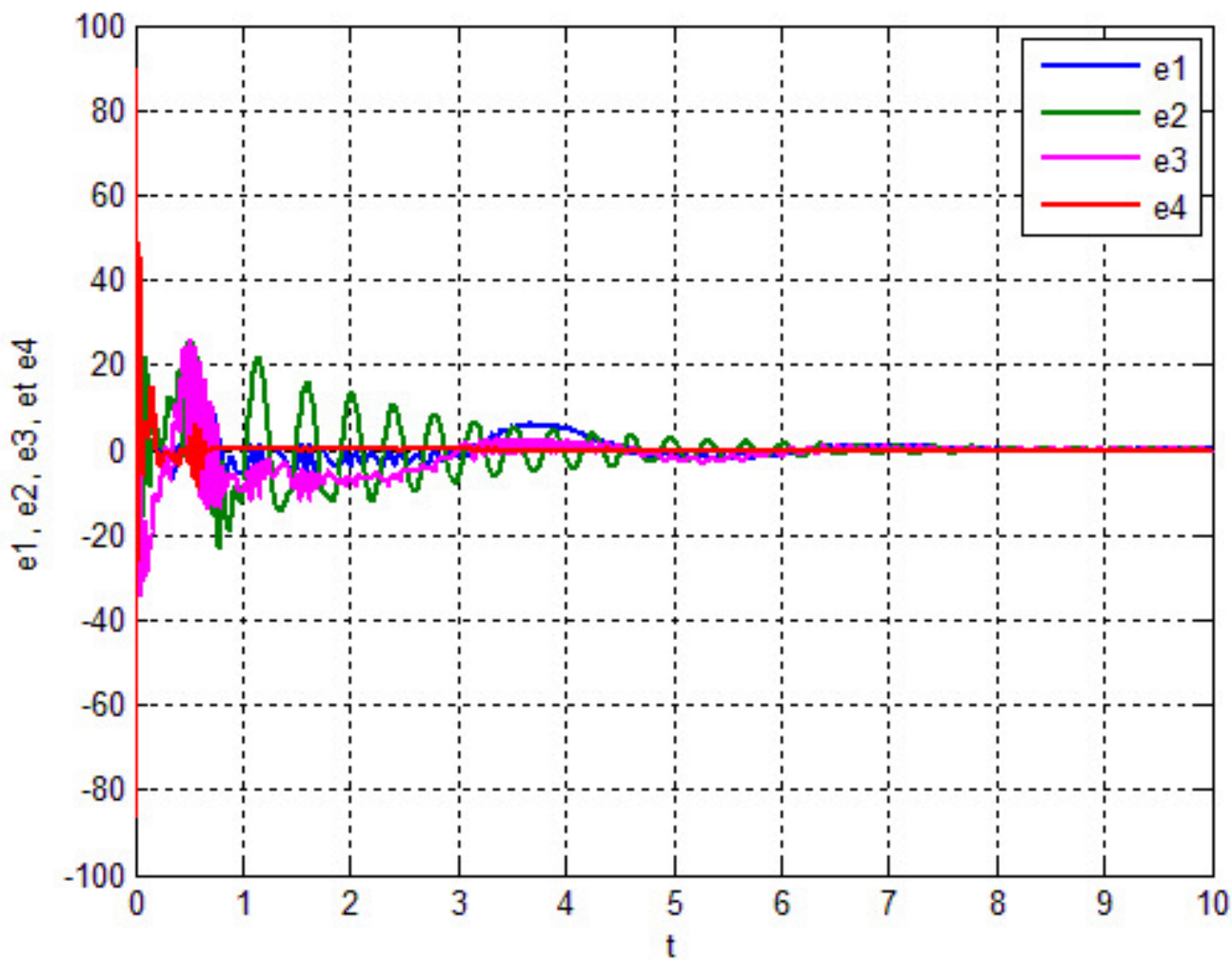

Fig. 2: Temporal history of the hybrid synchronization error of non identical systems (14) and (15).

\section{Conclusion}

In this paper, we have considered the stabilization and the hybrid synchronization for some non-identical financial hyperchaotic systems with continuous time according to the adaptive control method with unknown parameters. Numerical simulations are used to validate the obtained results via the approach considered for this kind of synchronization.

\section{Competing interests}

The authors declare that they have no competing interests. 


\section{Authors' contributions}

All authors have contributed to all parts of the article. All authors read and approved the final manuscript.

\section{References}

[1] O. Edward: Chaos in dynamical systems. Cambridge university press, 2002.

[2] . Fujisaka, T. Yamada: Stability theory of synchronized motion in coupled-oscillator systems. Progress of Theoretical Physics, 69(1), (1983), 32-47.

[3] L.M. Pecora, T.L. Caroll: Synchronization in chaotic systems. Physical Review Letters. 64, 821-825, (1990).

[4] C. Grebogi, Y. C. Lai: Controlling chaotic dynamical systems. Systems and control letters. 31(5), (1997), 307-312.

[5] H. Adloo, M. Roopaei: Review article on adaptive synchronization of chaotic systems with unknown parameters. Nonlinear Dynamics. 65(1), (2011), 141-159.

[6] H.K. Khalil: Nonlinear Systems. New York, Prentice Hall, 2002.

[7] W. Hahn: The Stability of Motion Springer, New York, USA, 1967.

[8] S. Vaidyanathan, C. Vollos, V. Pham, K. Madhavan: Analysis, adaptive. control and synchronization of a novel 4-D hyperchaotic hyperjerk system and its SPICE implementation. Archives of Control Sciences, 25(1), (2003), 135-158.

[9] 3. Hu, J., Chen, S., \& Chen, L. (2005). Adaptive control for anti-synchronization of Chua's chaotic system. Physics Letters A, 339(6), 455-460.

[10] Vaidyanathan, S. Vollos, C. (2015). Analysis and adaptive control of a novel 3-D

[11] conservative noequilibriumchaotic system Archives of Control Sciences, 25(3), 333-353.

[12] Hannachi, F. (2019). Analysis, dynamics and adaptive control synchronization of a novel chaotic 3-D system. SN Applied Sciences, 1(2), 158. 